\title{
Relations between Elder Abuse, Ageism and Perceptions of Age
}

\author{
Margarida Pedroso de Lima ${ }^{1 *}$, Maria Emília Vergueiro ${ }^{1}$, António-José Gonzalez², Paulo \\ Martins $^{3}$, João Garrido Oliveira ${ }^{1}$ \\ ${ }^{1}$ Faculty of Psychology and Educational Sciences, University of Coimbra \\ ${ }^{2}$ ISPA - Instituto Universitário de Ciências Psicológicas, Sociais e da Vida \\ ${ }^{3}$ Faculdade de Motricidade Humana, Universidade de Lisboa, Portugal
}

Centro de Investigação em Neuropsicologia e Intervenção Cognitivo Comportamental - CINEICC

UID/PSI/00730/2013

\begin{abstract}
*Corresponding Authors:Margarida Pedroso de Lima, Faculty of Psychology and Educational Sciences, University of Coimbra, Portugal
\end{abstract}

\begin{abstract}
The purpose of this study is to understandif the Ageismand perceptions of age of a group of elderly people is related with their experiences of being abused, mistreated or suffer from negligence.

33 older subjects were assessed with QEEA - Questions to Elicit Elder Abuse, Ageism Survey and POA Perceptions of Aging. The findings show some evidence of the correlations between discrimination, perceptions of ageingand abuse.

A prevention program designed to prevent abuse and based in active and participative methodologies-The Transparent Hands project-is presented as a tool to help and overcome the attitudinal resistances to change preconceptions of age.
\end{abstract}

Keywords:Ageism, Elder Abuse, Perceptions of Ageing, Prevention, Creative Action Methods, Role-Play.

Translational significance:

- Prevention programs are essential when dealing with elder abuse.

- Active and participative methodologies have big impact in changing attitudes.

- $\quad$ Theatre is a very powerful tool to give information, involve people and rehearsenew roles.

\section{INTRODUCTION}

\subsection{Elder Abuse and Neglect}

The social and technological changes triggered by the industrial revolution, the baby boom (Nelson, 2005), the progresses of science and medicine and the decrease of the birth rate changed the demographic map of the $20^{\text {th }}$ century. This new reality of demographic aging posed many different challenges and problems to contemporary societies (Simões, 2006) such as the increment of ageism and elder abuse.

Ageism is a systematic stereotyping and discrimination process against people because of their age (Butler, 1995). It is considered the third social "ism", after racism and sexism. Nevertheless, ageism is different due to the fact that everybody is vulnerable to it, since the majority of people will live long enough to reach the last stage of life (Palmore, 2001).

In the Second World Assembly on Aging of the United Nations, in 2002, the Commission to Social Development defined ageism as "one means by which the human rights of older persons are denied or violated. Negative stereotypes and denigration of older individuals can translate into lack of societal concern for older persons, risk of marginalization and denial of equality of access to opportunities, resources and entitlements" (Viegas \& Gomes, 2007, p. 29). In our society some beliefs and stereotypes against older people are rooted and commonly accepted, like rigidity/inflexibility, religiosity, low attractiveness, senility, unproductiveness, illness, difficulty in coping, poverty, 
asexuality, misery, dependence, conservatism, uniformity, isolation/loneliness, in identity crisis, with low self-esteem, difficulties in adapting to new roles and places, low motivation for the future, childishness, tendency to somatization, hypochondria, depression, suicide (Oliveira, 2008) and death. These prejudices can appear in a huge variety of contexts: institutions (Bytheway, 2005), health care services (Cuddy, Norton,\& Fiske, 2005), psychiatric contexts (Nelson, 2005; Nussbaum, Pitts, Huber, Krieger, \& Ohs, 2005), at work (Roscigno, Mong, Byron,\& Tester, 2007) and in everyday life.

Perceptions of aging, attitudes and decisions towards old people, national and international resources allocated to older people, are influenced by these stereotypes, prejudices and beliefs about the aging process (Bytheway, 2005; Burnett, 2014).

The mistreatment, neglect and elder abuse may be described as "the physical, emotional or psychological abuse of an older person by a formal or informal care provider. The abuse is repeated and is the violation of a person's human and civil rights by an individual or individuals who have power over the life of a dependant" (Eastman, 1984, Decalmer \& Glendenning, 1997, p. 1). This phenomenon occurs "within any relationship where there is an expectation of trust, which causes harm or distress to an older person" (Action on Elder Abuse, 1995; Decalmer \& Glendenning, 1997, p. 1). The National Centre on Elder Abuse (1998) defines seven kinds of elder abuse to noninstitutionalized people: physical, sexual, emotional/psychological, financial or material exploitation, abandonment, neglect, and self-neglect. However, there are other types as rights violation, for instance, privacy, confidentiality, free choice, religious freedom, freedom to refuse taking some psychotropic drugs, and free consent to be institutionalized, and kidnapping, which includes taking the elderly to institutions without their consent, preventing them from returning home using force, coercion or under illegitimate influence (Nerenberg, 2008).

According to some authors these are often sub referenced, as victims fear losing their caregivers, be alone, be placed in an institution, lose privacy and family relations, suffer recriminations, public display or to be held responsible by abusive behaviour (Ferreira-Alves \& Novo, 2006).

We also know, according to the National Elder Abuse Incidence Study (1998) that neglect is the most common type of abuse of the elderly and sons and daughters are the main perpetrators and which are the risk factors associated to the caregiver and the victim. We also know the consequences that abuse can have on older people, including weight loss, dehydration and malnutrition, emotional indifference, lack of interest in personal hygiene, indifference, inability to perform certain activities previously carried out autonomously, and alienation. Mello (2012) emphasizes that it may lead to increased mortality rates, reduce the years of productive life, increase on hospital care needs and canalso cause irreversible consequences for the victims.

Ageism brings a negative view of the older people and a negative perception of the aging process and can be a risk factor, not only of avoidance, denial or subordination behaviours (Sheets, 2005) but also of mistreatment, neglect or elder abuse situations.

A deeper knowledge of the stereotypes rooted in a specific population, and the prevalence and phenomenological nature of elder abuse episodes is fundamental to develop the means of primary prevention of this kind of situations. This type of intervention should be prioritized due to the fact that opens paths to promoting wellbeing and quality of life and it leads to lesser costs in public health.

\subsection{Primary Prevention}

In our society, the abusive and unfair treatment of the elderly is a growing phenomenon that urges to be responded effectively and, above all, prevented. To have impact, prevention actions must go beyond mere information. For this it is necessary to develop prevention programs specifically designed for old age and using active and participatory methodologies.

As we know, the aging of the population is a worldwide phenomenon. The underlying challenge involves dealing with an increasing number of older people whom, for historical, political and social circumstances, are more vulnerable: less educated, poorer, less social power, less active and less healthy. This state of vulnerability makes this age group become targets of discrimination and therefore abuse.

Due to the increase of cases of violence against the elderly, there has been a crescent interest in promoting a set of measures in order to prevent the different forms of abuse. These measures are important because they warn and assist in the detection and early intervention and hence the 
avoidance of these forms of violence. Thus, there are three forms of prevention: primary, secondary and tertiary (Costa, Pepper, Fights, Santos, \& Almeida, 2009).

Primary prevention aims to prevent the emergence of ill-treatment, through the identification of the causes and risk factors of abuse. It can also be enhanced through promotion of elder's and caregivers' skills and in the training of professionals through awareness-raising actions aimed at society and / or the promotion of active aging (Paulos, 2007).The studies highlight a growing consensus across studies regarding the extent and causes of mistreatment of older individuals in our society, as well as the urgent need for efforts to make mistreatment prevention programs for this population more effective and evidence based (Pillemer, Burnes, Riffin \&Lachs, 2016; Alon \&Berg-Warman, 2014).

As for the secondary prevention, it is intended to reduce the prevalence of abuse, or to identify and intervene early in order to avoid more serious consequences or even the recurrence of these cases. Therefore, these goals can be achieved by detection of abuse and / or risk factors, support for carers, as well as continuing education for caregivers.

Finally, with regard to tertiary prevention, it is the reduction of the consequences of a possible situation of abuse that is aimed. Seeking to minimize the damage and at the same time providing a better quality of life for the elderly through treatment and rehabilitation, with medical, psychological, social, legal and economic aids, as well as through treatment and rehabilitation of the offender (psychotherapeutic interventions) (Costa, Pepper, Fights, Santos, \& Almeida, 2009).

As stated by the UN Human Development Report (2000) Human development is a process of enhancing human capabilities, to enlarge the scope of choices and opportunities so that each person can lead a life of respect and value (UNDP,2000). In the UN Human Development Report (2014)it is emphasized the importance of including the paradigms of human development and human rights in the promotion of well-being. We believe that primary prevention is the path to make this possible.

Ageism brings a negative view of the older people and a negative perception of the aging process, which can be a risk factor, not only of avoidance, denial or subordination behaviors (Sheets, 2005) but also of mistreatment, neglect or elder abuse situations.

In this study we start to present a study about the relations between ageism, perceptions of aging and elder abuse. Finally we will present a program for primary prevention of elder abuse.

\section{METHOD}

\subsection{Participants}

Thirty-three elderly living in the community in the Northern Centre of Portugal took part in this study. Regarding to the socio-demographic characteristics of this sample, the average age was 77.21 $(S D=6.39)$, the majority were women $(\mathrm{N}=23 ; 69.7 \%)$ and widowers $(42.4 \%)$, followed by married $(36.4 \%)$, single $(15.2 \%)$ and divorced $(6.1 \%)$. The predominant educational level was primary school, followed by the illiterate $(27.3 \%), 6.1 \%$ completed 6 years of school and the secondary school and just $3 \%$ of the participants finished the ordinary level and the advanced level. The most prevalent socioeconomic level was the lowest $(78.8 \%)$, and the other participants had a medium level $(21.2 \%)$. The majority lived in their own house (75.8\%). As far as health is concerned, in this sample, $72.7 \%$ $(\mathrm{N}=24)$ didn't have any psychological problems, $97 \%$ had physical problems, no one was addicted to alcohol, drugs and non-prescribed medicine, $18.2 \%$ needed some help for daily life activities.

\subsection{Instruments and Procedure}

The instruments used in this research were:

- Questions to Elicit Elder Abuse(QEEA) originally validated from Carney, Kahan \& Paris(2003)and adapted to Portuguese by Ferreira-Alves \& Sousa(2005).The QEEA it is a screening and detection instrument for indicators of neglect and elder abuse; it comprises 15 items/questions that focus 4 kinds of elder abuse: physical, emotional, neglect and financial abuse and respondents have to answer if they have or not experienced each of them.

- Ageism Survey(AS) originally validated from Palmore (2001) and adapted to Portuguese by Ferreira-Alves \& Novo, (2006). The AS is a self-response questionnaire about ageism. It has 20 items, each one representing an example of negative personal or institutional stereotype, attitude and discrimination against elderly people. For each item, the subject must respond to 
how often he has experienced each of the episodes listed, marking the number of one of three possible responses: (0) never; (1) once; (2) more than once (Ferreira-Alves \& Novo, 2006). All reported episodes are related to negative forms of ageism, ie with negative discrimination (Palmore, 2001). It is possible to add to this instrument an index (AS_Total) calculated from the sum of the scores that the subject assigned to the different items.

- Perceptions of Aging (POA)was originally built by Löckenhoff, et al.(2004) and adapted to Portuguese by Lima(Löckenhoff, et al., 2004). This questionnaire was built to illustrate the perception of the changes of some characteristics (physical attractiveness, respect received from the others, general knowledge, social interaction, authority in family, ability to everyday tasks, wisdom, health, life satisfaction, ability to learn new information) throughout the aging process that the subject rates on a Likert scale ranging from 1 "decreases very much" to 5 "increases very much". It includes also two items: one about the frequency of contact with older people, and the other related to the participants' views about the way society sees the aging process.

After consent, the protocol was individually administered to each participant by the interviewer and the interviews took place in a calm, safe, and confidential environment.

\subsection{Data Analysis}

Data were analyzed using SPSS 21.0 (SPSS Inc. Chicago IL). The descriptive statistics was performed calculating means and standard deviation both for each item and the total scorefor each construct. TheSpearman coefficient, used due to the size of the samples,was calculated to assess the correlations between each construct of the three instruments. The statistical significance was assumed at a .05 level.

\section{RESULTS}

The results of Questions to Elicit Elder Abuse showed that $66.66 \%$ of the participants $(\mathrm{N}=22)$, presented at least an indicator of abuse. The most prevalent kind of elder abuse was emotional abuse $(\mathrm{N}=17 ; 72.7 \%)$, followed by neglect $(\mathrm{N}=9 ; 40.9 \%)$ and financial abuse $(\mathrm{N}=5 ; 22.7 \%)$. In this sample there were no cases of physical abuse reported.

Fifteen older people (45.5\%) answered that they had never experienced the situations illustrated by the items of Ageism Survey. In this questionnaire, the most experienced item was item 12 (A doctor or nurse assumed my ailments were caused by my age). The second most reported item was item 17 (Someone assumed I could not understand because of my age). In the AS_Total (the index that represents the sum of the scores of all the items of the Ageism Survey by a participant) the average was 2.15 ( $S D=5.24)$. The lowest result was zero, and the highest 30.

About the Perceptions of Aging (POA) the results are summarized in the Table 1.

Table1.Mean and standard deviation of the results in the Perceptions of Aging

\begin{tabular}{|l|l|l|}
\hline \multicolumn{1}{|c|}{ Items } & \multicolumn{1}{c|}{ M } & \multicolumn{1}{c|}{ SD } \\
\hline 1. Physical Attractiveness & 2.18 & 0.81 \\
\hline 2. Respect Received & 2.97 & 0.73 \\
\hline 3. General Knowledge & 2.88 & 1.05 \\
\hline 4. Social Interaction & 2.73 & 0.94 \\
\hline 5. Authority in Family & 2.91 & 0.68 \\
\hline 6. Ability to Everyday Tasks & 2.30 & 0.73 \\
\hline 7. Wisdom & 2.88 & 0.99 \\
\hline 8. Health & 1.73 & 0.72 \\
\hline 9. Life Satisfaction & 2.58 & 0.9 \\
\hline 10. Ability to Learn New Informations & 2.39 & 1.03 \\
\hline 11. Contact with Older People & 4.79 & 0.86 \\
\hline 12. Perception of the Portuguese People About Aging & 2.55 & 1.23 \\
\hline
\end{tabular}

The majority of the interviewed referred having contacts with other older people almost every day and they believed that the Portuguese have a negative view of the aging process (see item 12). Every item of POA was rated as decreasing with age. Health was the characteristic considered decreasing more with age $(\mathrm{M}=1.54 ; S D=0.69)$ and the respect received, the one that was kept more stable throughout life $(\mathrm{M}=2.97 ; S D=0.73)$. 
To achieve the initial aim of our study, we correlated the number of indicators of the four kinds of elder abuse represented on Questions to Elicit Elder Abuse (QEEA) its sum of total indicators (QEEA_Total) and the sum of total items of the Ageism Survey (AS). However, no correlations were found (Table 2).

Table2.Correlations between the scores of QEEA and the score of $A S(N=33)$

\begin{tabular}{|c|c|c|c|c|}
\hline Items & \multicolumn{3}{|c|}{ QEEA } \\
\hline & Em & Ng & Fn & Total \\
\hline $\mathbf{A S}$ & .207 & .200 & -.279 & .136 \\
\hline
\end{tabular}

The inexistence of correlations was also the pattern found with the scores of the 12 items of the POA andthe QEEA items.

So, new correlations were done between the same scores of QEEA and the 20 items of Ageism Survey.

Table3. Correlations between the scores of QEEA and $A S(N=33)$

\begin{tabular}{|c|c|c|c|c|}
\hline Items & \multicolumn{4}{|c|}{ QEEA } \\
\hline AS & Em & Ng & Fn & Total \\
\hline 4 & $.372^{*}$ & $.531^{* *}$ & -.107 & $.423^{*}$ \\
\hline 9 & .220 & $.370^{*}$ & -.075 & .281 \\
\hline 10 & .215 & $.454^{*}$ & -.107 & .320 \\
\hline 11 & .220 & $.370^{*}$ & -.075 & .281 \\
\hline 13 & .218 & $.456^{* *}$ & -.107 & .322 \\
\hline 14 & .220 & $.370^{*}$ & -.075 & .281 \\
\hline 15 & .220 & $.370^{*}$ & -.075 & .281 \\
\hline 18 & .220 & $.370^{*}$ & -.075 & .281 \\
\hline 19 & .220 & $.370^{*}$ & -.075 & .281 \\
\hline
\end{tabular}

$* p \leq .05 ; * * p \leq .01$

The results in the Table 3 shows the existence of a medium positive correlation between the indicators of emotional abuse and the item 4 of AS ( $I$ was called an insulting name related to my age): $r_{\mathrm{s}}=0.372$; $\mathrm{N}=33 ; \mathrm{p}=0.033$. So, whenever this kind of ageism was verified, high values of emotional abuse indicators will appear.

A positive correlation between neglect indicators and some items of Ageism Survey were also found. A strong correlation was found with item 4 [(I was called an insulting name related to my age $)$; $\left.r_{\mathrm{s}}=0.531 ; \mathrm{N}=33 ; \mathrm{p}=0.001\right]$ and some medium correlations were identified with the other items: $9[(I$ was rejected as unattractive because of my age $\left.) ; r_{\mathrm{s}}=0.370 ; \mathrm{N}=33 ; \mathrm{p}=0.034\right], 10$ [(I was treated with less dignity and respect because of my age $\left.) ; r_{\mathrm{s}}=0.454 ; \mathrm{N}=33 ; \mathrm{p}=0.008\right], 11$ [(A waiter or waitress ignored me because of my age $\left.) ; r_{\mathrm{s}}=0.37 ; \mathrm{N}=33 ; \mathrm{p}=0.034\right], 13$ [(I was denied medical treatment because of my age $\left.) ; r_{\mathrm{s}}=0.456 ; \mathrm{N}=33 ; \mathrm{p}=0.008\right], 14$ [(I was denied employment because of my age $)$; $\left.r_{\mathrm{s}}=0.37 ; \mathrm{N}=33 ; \mathrm{p}=0.034\right], 15$ [(I was denied a promotion because of my age $) ; r_{\mathrm{s}}=0.37 ; \mathrm{N}=33$; $\mathrm{p}=0.034$ ], 18 [(Someone told me, "You're too old for that."); $\left.r_{\mathrm{s}}=0.37 ; \mathrm{N}=33 ; \mathrm{p}=0.034\right]$ e 19 [(My house was vandalized because of my age); $\left.r_{\mathrm{s}}=0.37 ; \mathrm{N}=33 ; \mathrm{p}=0.034\right]$. These results shows that the increase of neglect indicators is related to an increase of social discriminating situations in old age.

The QEEA_Total index is moderately positively correlated with item 4 of AS [ $($ w was called an insulting name related to my age); $\left.r_{\mathrm{s}}=0.423 ; \mathrm{N}=33 ; \mathrm{p}=0.014\right]$. So, the increase of the total number of abuse indicators is related to the increase of the experience of these discriminating situations.

Regarding the correlations between the POA and the QEEA, the indicator of neglect appears moderately and negatively correlated with the items 3 and 9 of POA: 3-general knowledge $\left(r_{\mathrm{s}}=-0.401\right.$; $\mathrm{N}=33 ; \mathrm{p}=0.021)$; 9-life satisfaction $\left(r_{\mathrm{s}}=-0.404 ; \mathrm{N}=33 ; \mathrm{p}=0.02\right)$. That is, a high number of neglect indicators are related to the perception that the general knowledge and life satisfaction decrease as the aging process happens.

The correlations (Table 4) between the items of POA and the items of the AS show the decreasing of some characteristics with age is strongly or moderately correlated with the experience of some ageist situations described in the items of Ageism Survey. The POA's item correlated with more AS's items was item 5 (authority in family). This POA's item was correlated with 12 of 20 AS's items [2 (I was sent a birthday card that pokes fun at old people), 3 (I was ignored or not taken seriously because of 
my age), 4 (I was called an insulting name related to my age), 5 (I was patronized or "talked down to" because of my age), 9 (I was rejected as unattractive because of my age), 10 (I was treated with less dignity and respect because of my age), 11 (A waiter or waitress ignored me because of my age), 13 (I was denied medical treatment because of my age), 14 (I was denied employment because of my age), 15 (I was denied promotion because of my age) and 19 (My house was vandalized because of my age)]. The item 2 of POA (respect received), is correlated with seven items of Ageism Survey [2 (I was sent a birthday card that pokes fun at old people), 9 (I was rejected as unattractive because of my age), 11 (A waiter or waitress ignored me because of my age), 14 (I was denied employment because of my age), 15 (I was denied promotion because of my age), 18 (Someone told me, "You're too old for that.") and 19 (My house was vandalized because of my age)]. The item 10 of POA (Ability to learn new information) is correlated with 3 items of AS [3 (I was ignored or not taken seriously because of my age), 5 (I was patronized or "talked down to" because of my age) and 13 (I was denied medical treatment because of my age). The fourth item of POA (social interaction), is correlated with items 3 (I was ignored or not taken seriously because of my age) and 4 (I was called an insulting name related to my age) of AS. The items 9 (life satisfaction) and 11 (contact with older people) of Perceptions of Aging are correlated with item 16 (Someone assumed I could not hear well because of my age).

Table4. Correlations between the scores in POA and AS items $(N=33)$

\begin{tabular}{|l|c|c|c|c|c|c|c|}
\hline \multirow{2}{*}{ POA } & \multicolumn{7}{|c|}{$\mathbf{A S}$} \\
\cline { 2 - 8 } & $\mathbf{2}$ & $\mathbf{4}$ & $\mathbf{5}$ & $\mathbf{6}$ & $\mathbf{9}$ & $\mathbf{1 0}$ & $\mathbf{1 1}$ \\
\hline AS1 & -.235 & -.100 & -.212 & $.345^{*}$ & -.075 & -.122 & .080 \\
\hline AS2 & $-.383^{*}$ & -.295 & $-.367^{*}$ & .193 & -.280 & -.264 & .045 \\
\hline AS3 & .007 & $-.375^{*}$ & $-.622^{*}$ & -.236 & -.068 & $-.472^{* *}$ & .080 \\
\hline AS4 & -.283 & $-.423^{*}$ & $-.487^{*}$ & .079 & -.150 & -.243 & .064 \\
\hline AS5 & -.092 & -.062 & $-.408^{*}$ & -.253 & .148 & $-.362^{*}$ & .080 \\
\hline AS9 & $-.383^{*}$ & -.295 & $-.367^{*}$ & .193 & -.280 & -.264 & .045 \\
\hline AS10 & 009 & -.332 & $-.528^{*}$ & -.073 & -.142 & $-.379 *$ & .064 \\
\hline AS11 & $-.383^{*}$ & -.295 & $-.367^{*}$ & .193 & -.280 & -.264 & .045 \\
\hline AS13 & -.008 & -.334 & $-.528^{* *}$ & -.062 & -.150 & $-.379 *$ & .064 \\
\hline AS14 & $-.383^{*}$ & -.295 & $-.367^{*}$ & .193 & -.280 & -.264 & .045 \\
\hline AS15 & $-.383^{*}$ & -.295 & $-.367^{*}$ & .193 & -.280 & -.264 & .045 \\
\hline AS16 & -.275 & -.028 & -.256 & .073 & $-.403 *$ & -.084 & $-.451 * *$ \\
\hline AS18 & $-.383^{*}$ & -.295 & $-.367 *$ & 193 & -.280 & -.264 & .045 \\
\hline AS19 & $-.383^{*}$ & -.295 & $-.367^{*}$ & 193 & -.280 & -.264 & .045 \\
\hline AS_Total & .090 & .118 & -.142 & .207 & -.041 & -.106 & -.185 \\
\hline
\end{tabular}

$* p \leq .05 ; * * p \leq .01$

The index AS_Total is moderately and negatively correlated with Mini Mental State Examination $\left(r_{\mathrm{s}}=-0.381 ; \mathrm{N}=33 ; \mathrm{p}=0.029\right)$ which means that, the better the cognitive state of the individuals of our sample, the less prone to ageist episodes he/she will be.

\section{DISCUSSION}

Ageism as a negative view of the elderly influences social representations, beingdescribed in the literature as a risky factor to elder abuse (Lachs, 1995; Nelson, 2005). The Risk model of elder mistreatment in domestic settings (National Research Council, 2003) reinforces this vision, emphasizing that beliefs and attitudes that both older people and their caregivers have about aging, will influence the way they interact with each other. One of the consequences of this interaction can be elder abuse.

Our study was designed to understand the relationships between these two phenomena, hoping to find a positive relation between the number of indicators of abuse identified by older people and the discriminating behaviors to which they are submitted. However, comparing the indexes of Questions to Elicit Elder Abuse (QEEA) with the index of the sum of scores of the Ageism Survey (AS_Total), no correlation was found. Nevertheless, once ageism comprises such a wide variety of stereotypes, prejudices and discriminating behaviors, it became relevant to correlate each one of the items of AS with QEEA's indexes, trying to find some specific behaviors or attitudes which were effectively related to the elder abuse's indicators. 
The results show that some items of Ageism Survey (namely items 4, 9, 10, 11, 13, 14, 15, 18 e 19) are positively correlated with QEEA indexes. This shows that the more indicators of abuse older people mark the more prone they will be to ageist behaviors.

The fact that item 4 of AS (I was called an insulting name related to my age) is the one which appears with the highest correlation with the indexes of emotional abuse and neglect and the QEEA_Total, suggests that this discriminating situation is felt as particularly abusive by older adults, due to a penchant for disrespect and the decrease of older people status. The disrespect also implies some stereotypes about ageing, from senility, loss of capacities, cognitive impairment, decrease of attractiveness, dependence on someone else, and it can contribute to the decrease of self-esteem. Hence this ageist situation is felt as particularly relevant and is related to some kinds of elder abuse. The correlation between the indicators of neglect and items 10 (I was treated with less dignity and respect because of my age) and 18 (Someone told me, "You're too old for that.") can be seen in the same way.

The remaining items appear correlated with neglect indicators. A greater relevance should be given to the correlation between these indicators and item 13 (I was denied medical treatment because of my age). This item of Ageism Survey is correlated with the stereotypes concerned with somatization, hypochondria, depression, senility and also the belief that some health problems and symptoms are simply due to aging. These stereotypes and beliefs influence attitudes towards the elderly, and also contribute to a devaluation of complaints and symptoms presented by them, which can originate faults of communication that severely constrain the clinical status of older people. In extreme cases it can also put the person in risk, and it is one of the highest causes of neglect in health contexts (Nussbaum et al., 2005).

One possible explanation for no significant correlations between the indexes of Questions to Elicit Elder Abuse and Ageism Survey, is the fact that some items of both instruments had never been experienced by the participants.

The results with QEEA show that the most prevalent kind of abuse was emotional abuse, followed by neglect. These results are consistent with those found by Fernandes \& Dionísio (2009) in a sample of 131 older people from the municipalities of Vouzela and Coimbra (Portugal), but they counter the data of Ferreira-Alves \& Sousa (2005) and National Elder Abuse Incidence Study (NCEA, 1998), where neglect emerged as the most prevalent elder abuse category, followed by the emotional/psychological abuse.

In this study, $54.5 \%$ of the subjects reported had already lived one or more situations portrayed by Ageism Survey's items. These outcomes reflect some stereotypes possibly rooted in the Portuguese culture, especially among health professionals (whose importance and consequences were explained above). The stereotype of senility seems also rooted.

The existence of some negative correlations between AS items and the perception of some characteristics with aging, suggests a mutual influence between stereotypes/discriminating attitudes of the Portuguese society and a negative perception by older people about aging, once all characteristics present in Perceptions of Aging are portrayed by them as suffering a decrease with aging. Although the authority in family is not identified as the most declining item, this is the feature that appears correlated with more items of Ageism Survey (12), which can give clues to the fact that some of these ageist attitudes are happening in families, the place where they are particularly harmful. At the same time, some of the discriminating behaviors are correlated with a perception of decline of the respect received, which suggests that these attitudes are also felt as injurious.

On the one hand, the existence of some correlations between indicators of neglect and some items of Perceptions of Aging (life satisfaction and general knowledge) suggest that the existence of these indicators contribute to a feeling of lesser life satisfaction; on the other hand it shows that the feeling of decrease of the general knowledge can cause a feeling of dependence on other people (who have more knowledge) and, as an outcome, more abuse.

\subsection{The Transparent Hands Project}

With the aim of raising awareness of the population towards elder abuse a primary prevention project was developed. The "Transparent Hands" project gives information about the types, causes, risk 
factors and consequences of elder abuse at the same time that, through active methods (Noice, Noice \& Kramer, 2014), invites people to reflect and develop assertiveness about this issue. The "Transparent Hands" aims to contribute to awareness and community information for the problem of abuse and ill-treatment against the elderly at the same time empowers the participants. It is important not only to clarify and raise awareness among potential "victims" as well as the current or future caregivers, family members and all professionals who interact and support the elderly. In this way, we intend with this project to cover the largest number of people taking advantage of the time people spend in the waiting rooms of the health care centres.

Through direct role-play of abuse behaviours the dynamics of people relationships in this realm are explored. The "Transparent Hands" appears in the context of artistic/creative action methods and to respond to the call to empower and give tools for participation and social intervention of the population (Raeanne et al., 2016). The scientific literature on the enhancement of healthy aging and in the prevention of elderly abuse through active participation through artistic methodologies is an underinvestigated corner of aging research (Noice, Noice \& Kramer, 2014). Nevertheless,Noice, Noice and Kramer (2014) emphasize that studies concerning different arts like dance, expressive writing, music (singing and instrumental), theatre arts, and visual arts encounter many mental/physical benefits from memory to quality of life.

The project "Transparent Hands" has theoretical influences in Theatre of the Oppressed of Augusto Boal and in the Psychodrama of Jacob Moreno. Moreno followed the idea of Aristotle that people who watched a scene felt identified and eventually had a catharsis. If this passive catharsis happened, Moreno put the hypothesis that a more active one may happen if the viewer would accept to be an actor in the drama of his own life (see Zerka's Moreno article "Beyond Aristotle, Breuer and Freud: Moreno's contribution to the concept of catharsis, in Moreno, 2006). This idea has been validated and psychodrama was recognised as a method of development, treatment and intervention. Abuse interferes with the spontaneity conceived as the energy needed to cope with the changes of our life. Psychodrama techniques were conceived to promote spontaneity and gain flexibility in role-taking.

The Theatre of the Oppressed is an aesthetic means to help people to analyse their past, in the context of their present, and subsequently (re)invent their future, without waiting for it. Theatre of the Oppressed is rehearsal for reality. The oppressed are those individuals or groups who are socially, culturally, politically, economically, racially, sexually, or in any other way deprived of their right to Dialogue or in any way impaired to exercise this right. Dialogue is defined as to freely exchange with others, as a person and as a group, to participate in human society as equal, to respect differences and to be respected. Because dialogues have the tendency to become monologues, which creates the relationship oppressors - oppressed, the main principle of Theatre of the Oppressed is to help restore dialogue among human beings.

Boal (1995, pp. 14-15), after stating that "theatre (...) is the true nature of humanity", defines the Theatre of the Oppressed as "a system of physical exercises, aesthetic games, image techniques and special improvisations whose goal is to safeguard, develop and reshape this human vocation, by turning the practice of theatre into an effective tool for the comprehension of social and personal problems and the search for their solutions".

The procedure used in Transparent Hands consists on presenting role-plays of elder abuse scenes and then ask the public to discuss and solve the scenes. The recreation of scenes based in true stories ${ }^{1}$ that were gathered through interviews of people who suffered abuse, scope the various types of abuse and the risk factors of the abuser and the victim. Some examples of scenes: 1) Financial AbuseScene Addicted grandson steals money from sleeping and ill grandmother. While withdrawing money he expresses some stereotypes 'she no longer needs the money'; 'her life is over'; 'it has no interest for you but the money helps me'; 2) Physical Abuse Scene - Old mother with dementia is obliged to dress up quickly in the middle of pushing and slapping by the overstressed daughter; 3) Psychological Abuse Scene - Bedridden husband insults elderly wife because she is slow in bringing water; 4) Negligence Scene - Daughter doesn't care if her father with Alzheimer's Disease is taking his medicine correctly.

\footnotetext{
${ }^{1}$ This stories were collected in a qualitative research developed in Minho University by Ferreira-Alves (personal communication) with the intent of understanding the narratives of the people involved in elder abuse situations.
} 
At the end of each scene there is one question made to the abused elderly: 'How did you feel? '. The answer is always the same: 'Sad. Very sad'.

At the end of the presentation the presenter enquires the public: 'How did you feel?'. And the debate begins.

The animator of the performance wears transparent gloves (the image of the palm of the hands is a symbol of the fight against violence, abuse and oppression, the transparency states for the importance of not hiding more this reality) and interacts with the public (people present in the waiting room of health care centres) and leads them to reflect / discuss / be aware of what is abuse, its different manifestations, its causes, its consequences and implications. He also invites people to change roles with the victim(s) and rehearsal solutions to the problem.

In the end of the encounter, information is provided on how to do / act to prevent and intervene in situations of abuse of older people.

\section{CONClusions}

This study was designed to look for a relationship between ageism (index of AS Ageism Survey) and the risk of elder abuse (through the indexes of indicators of abuse of QEEA - Questions to Elicit Elder Abuse), and the relationship between these two phenomena and the perceptions of aging (POA Perceptions of Aging), in a sample of older people in the community.

No correlations between the two phenomena were found, when a global comparison was done between the index of AS, the items of POA and the indexes of elder abuse identified by the participants. However, a more detailed item analysis of the Ageism Survey allows finding out some ageist situations that were correlated with indicators of abuse and the perception of the decrease of some characteristics with age.

As these are two emerging phenomena in contemporary societies it is important to develop future research about them, with broader samples and representative studies of the population.

A deeper knowledge of the stereotypes rooted in the Portuguese population, and the prevalence and phenomenological nature of elder abuse episodes is fundamental to develop the means of primary prevention of this kind of situations. This intervention should be primarily developed due to the fact that it leads to less cost in public health, opening paths to promoting well-being, life satisfaction and quality of life throughout the life span.

The domain of abuse and neglect of the elderly population is morally appealing and although these last years the research has increased, the majority are studies of prevalence or simple description of associations between variables. But screening is only one step in the set of goals that should be made to understand and act on abuse in old age.Elder abuse and mistreatment is now recognized internationally as a pervasive and growing problem, urgently requiring the attention of health care systems, social welfare agencies, policymakers, and the general public. In this article, we provide an overview of global issues in the field of elder abuse, with a focus on prevention through active and artistic methodologies. Scientific validation of this procedure are needed. This data will be provided in the near future.

The first scientific article that focuses the abuse of an elderly person was described by Baker in 1975 . At that time, this phenomenon, identified by a doctor in the course of his practice, was described as a subject that deserved special clinical attention. However, this clinical focus on abuse issues was "aborted" leading to a predominance of legal and social concerns.

Preventive actions against abuse of elders began to be protective of vulnerable older people and, despite its good intentions, led often to ageism and paternalism (Nerenberg, 2008). Consequently, many technicians interested in changing these negative side effects have been emphasizing their commitment to customer autonomy, self-determination and personal freedom changing hence the paradigm that emphasized the protection for one that emphasizes power and resources of older people (Jaaniste, 2015).

This paradigm shift necessarily implies a different emphasis on procedures valued to prevent and cope with abuse and mistreatment, defending approaches that promote self-esteem, information, training 
skills and problem solving, such as peer groups, support groups, psycho-educational groups, psychological and legal counselling (Nerenberg, 2008).

All measures and interventions directed to accept the 'other' in its history and constraints and that believes that despite the obstacles, persons can still develop and update their potential, are based on the humanist assumptions well formulated by Carl Rogers (1961). All interventions aiming change in the other's wellbeing, conditions and self-esteem should be based on absolute respect, trying to promote and achieve empathy and congruence. We believe that the rational involving the two main theoretical and practical inspirations for the Transparent Hands project follow this humanistic baselines and stand up for the importance of more holistic interventions in this realm.

Acknowledging in depth the stereotypes present in the Portuguese population as well as the prevalence and phenomenological nature of the episodes of abuse is fundamental to develop the means of primary prevention of this type of situations. An intervention primarily directed to changing attitudes through active methodologies should be favored.

\section{REFERENCES}

[1] Alon, S., \&Berg-Warman, A. (2014). Treatment and prevention of elder abuse and neglect: Where knowledge and practice meet-A model for intervention to prevent and treat elder abuse in Israel. Journal of Elder Abuse \& Neglect, 26(2), 150-171. 10.1080/08946566.2013.784087.

[2] Baker, A.A. (1975). Granny-battering. Modern Geriatrics, 5, 20-24.

[3] Boal, A. (1995). The Rainbow of Desire. The Boal method of Theatre and Therapy. New York, Routledge.

[4] Burnett, J. (2014). Prevention and early identification of elder abuse. In Achenbaum W.A.,Murphy, K.P.Clinics In Geriatric Medicine, 30 (3), 743-59.10.1016/j.cger.2014.08.013.

[5] Butler, R. (1995). Ageism. In G. Maddox (Ed.), The encyclopedia of aging, 2nd ed., 38-39. New York: Springer Publishing Co.

[6] Bytheway, B. (2005). Ageism. In M. Johnson, V. Bengtson, P. Coleman, \& T. Kirkwood (Eds.), The Cambridge handbook of age and ageing, 338-345. Cambridge: University Press.

[7] Carney, M.T., Kahan, F.S., \& Paris, B. (2003). Elder abuse: Is every bruise a sign of abuse?. The mount sinai journal of medicine, 70: 2, 69-74.

[8] Cuddy, A., Norton, M., \& Fiske, S. (2005). This old stereotype: The pervasiveness and persistence of the elderly stereotype. Journal of Social Issues, 61(2), 267-285.

[9] Decalmer, P.,\& Glendenning, F. (1997). The mistreatment of the elderly people. London: Sage Publications.

[10] Fernandes, C. \& Dionísio, R. (2009). Violência na pessoa idosa. Trabalho realizado no âmbito do II Curso de Pós-Licenciatura de Especialização em Enfermagem Comunitária não publicado. Escola Superior de Enfermagem de Coimbra.

[11] Ferreira-Alves; J., \& Sousa, M. (2005).Indicadores de maus-tratos a pessoas idosas na cidade de Braga: estudo preliminar. Sociologia, 15, 303-313.

[12] Ferreira-Alves, J.,\& Novo, R. (2006). Evaluation of ageism in Portugal. International Journal of Clinical and Health Psychology, 6 (1), 65-77.

[13] Jaaniste, J., Linnell, S., Ollerton, R. L., \& Slewa-Younan, S. (2015). Drama therapy with older people with dementia-Does it improve quality of life? The Arts in Psychotherapy, 43, 40-48. 10.1016/j.aip.2014.12.010.

[14] Lachs, M.S. (1995). Preaching to the unconverted: Educating psysicians about elder abuse. Journal of Elder Abuse and Neglect, 7(4), 1-12.

[15] Löckenhoff, C. E. et al.,(2014). Gender Stereotypes of Personality: Universal and Accurate? Journal of Cross-Cultural Psychology 1-20. sagepub.com/journalsPermissions.nav. DOI: 10.1177/0022022113520075. jccp.sagepub.com.

[16] Mello, J.D.A., Durme, T.V., Macq, J., Declerq, A. (2012). Intervention to delay institutionalization of frail older persons: design of longitudinal study in the home care setting. BMC Public Health, 12, 615.

[17] Moreno, Z. (2006). The Quintessential Zerka: writings by Zerka Toeman Moreno on Psychodrama, Sociometry and Group Psychotherapy. New York, Routledge.

[18] National Center on Elder Abuse at the American Public Human Services Association. (1998). The national elder abuse incidence study. Grand Forks, ND: National Center on Elder Abuse. Retrieved fromhttp://www.aoa.gov/ eldfam/Elder_Rights/Elder_Abuse/ABuseReport_Full.pdf. 
[19] National Research Council (2003). Elder mistreatment: abuse, neglect, and exploitation in an aging America. Washington, D.C.: The National Academies Press.

[20] Nelson, T. (2005). Ageism: prejudice against our feared future self. Journal of Social Issues, 61(2), 207221.

[21] Nerenberg, L. (2008). Elder abuse prevention: emerging trends and promising strategies. New York: Springer Publishing Company.

[22] Noice, T., Noice, \& H., Kramer, A. F., (2014). Participatory Arts for Older Adults: A Review of Benefits and Challenges. The Gerontologist, 54 (5). 741-741.

[23] Nussbaum, J.F., Pitts, M.J., Huber, F.N., Krieger, J.L., \& Ohs, J.E. (2005). Ageism and ageist language across the life span: intimate relationships and non-intimate interactions. Journal of Social Issues, 61(2), 287-305.

[24] Pillemer, K.,Burnes, D.,Riffin, C.,\&Lachs, M.S. (2016). Elder Abuse: Global Situation, Risk Factors, and Prevention Strategies.The Gerontologist, 56(2).194-205. 10.1093/geront/gnw004.

[25] Moore, R. C., Straus, E., Dev, S. I., Parish, S. M., Sueko, S., \&Eyler, L.T.,(2016). Development and pilot randomized control trial of a drama program to enhance well-being among older adults. The Arts in Psychotherapy, 52, 1-9 http://dx.doi.org/10.1016/j.aip.2016.09.007

[26] Oliveira, J. B. (2008). Psicologia do envelhecimento e do idoso (3rd ed.). Porto: Livpsic.

[27] Palmore, E. (2001). The ageism survey: First findings. The Gerontologist, 41(5), 572-575.

[28] Rogers, Carl. (1961). On Becoming a Person: A Therapist's View of Rogers, Carl. (1970). On Encounter Groups. New York: Harrow Books.

[29] Roscigno, V., Mong, S., Byron, R., \& Tester, G. (2007). Age Discrimination, Social Closure and Employment. Social Forces, 86(1), 313-334.

[30] Sheets, D.J. (2005). Aging with disabilities: Ageism and more. Generations, 29, 37-41.

[31] Simões, A. (2006). A nova velhice. Porto: Ambar.

[32] UNDP, Human Development Report (2000). http://hdr.undp.org/sites/default/files/reports/261/hdr_2000_ en.pdf

[33] UNDP, Human Development Report (2014). Sustaining Human Progress: Reducing Vulnerabilities And Building Resilience Viii, http://hdr.undp.org/ sites/default/files/hdr14-report-en-1.pdf

[34] Viegas, S.M., \& Gomes, C.A. (2007). A identidade na velhice. Porto: Ambar.

AUTHORS' BIOGRAPHY

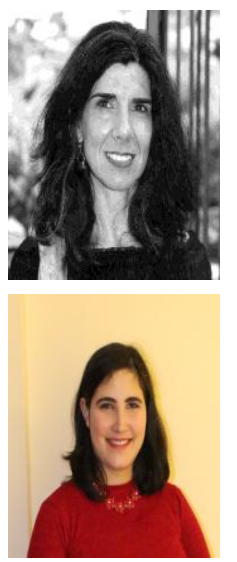

Margarida Pedroso de Lima - Associated Professor at the Faculty of Psychology and Educational Sciences of the University of Coimbra, Portugal. Responsible for Psychogerontology interventionin the Clinical and Health Psychology area of this Faculty. Psychotherapist with Specialization in Gestalt and in Psychodrama.

Maria EmíliaVergueiro- Specialist Psychologist in the Area of Clinical and Health Psychology and in Psychogerontology; Psychotherapist with Specialization in Constructivist Psychotherapies by the Portuguese Society of Constructivist Psychotherapies; With postgraduate degrees in Clinical Neuropsychology and in Neuropsychological Intervention.

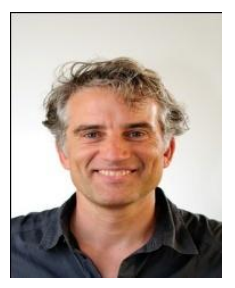

António Gonzalez Professor in ISPA (ISPA - InstitutoUniversitário de CiênciasPsicológicas, Sociais e da Vida). Psychotherapist with Specialization in Psychodrama.

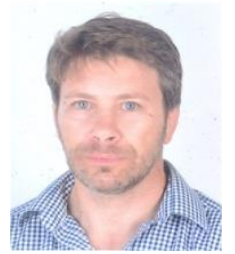

Paulo Martins Professor in the Faculty of Human Motricity, University of Lisbon, Portugal de Motricidade Humana, Universidade de Lisboa, Portugal. Researcher 


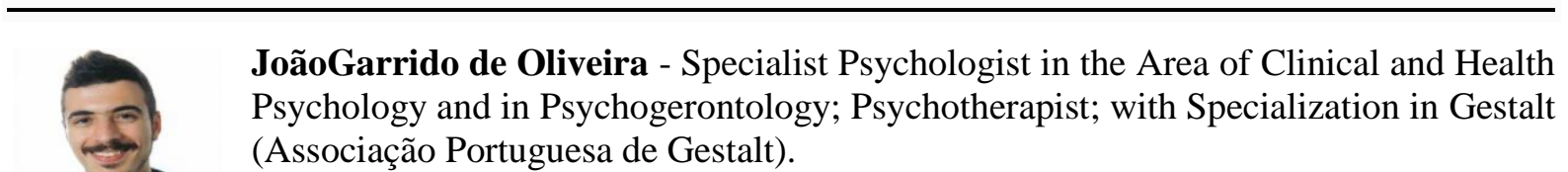

JoãoGarrido de Oliveira - Specialist Psychologist in the Area of Clinical and Health (Associação Portuguesa de Gestalt).

Citation: Margarida Pedroso de Lima, Maria Emília Vergueiro, António-José Gonzalez, Paulo Martins, João Garrido Oliveira. "Relations between Elder Abuse, Ageism and Perceptions of Age" International Journal of Humanities Social Sciences and Education (IJHSSE), vol 5, no. 6, 2018, pp. 91-102. doi: http://dx.doi.org/10. 20 431/2349-0381.0506012.

Copyright: () 2018 Authors. This is an open-access article distributed under the terms of the Creative Commons Attribution License, which permits unrestricted use, distribution, and reproduction in any medium, provided the original author and source are credited. 